\title{
Closed Geodesics on the Space of Stable Two-Monopoles ${ }^{\star}$
}

\author{
Larry Bates ${ }^{1}$ and Richard Montgomery ${ }^{2}$ \\ ${ }^{1}$ Department of Mathematics, University of Calgary, Calgary, Alberta, Canada T2N 1N4 \\ ${ }^{2}$ Department of Mathematics, Massachusetts Institute of Technology, Cambridge, MA 02138, \\ USA
}

\begin{abstract}
We show that the Atiyah-Hitchin metric on the space of stable twomonopoles admits closed geodesics.
\end{abstract}

\section{Introduction}

It is important to understand whether or not two monopoles of like magnetic charge can exist as an orbiting bound pair. In the context of the classical $S U(2)$ Yang-Mills-Higgs (YMH) equations, this problem was posed by Jaffe and Taubes in [1].

In 1981 Manton [2] suggested that for certain initial conditions, the YMH equations in the Bogolmon'yi-Prasad-Sommerfeld (BPS) limit may be approximated by geodesic flow on a manifold $\mathscr{M}_{2}$. The initial conditions are those of a pair of slow-moving BPS monopoles. The manifold $\mathscr{M}_{2}$ forms a non-compact fourdimensional manifold. The details may be found in Hurtubrise [3] or Donaldson [4]. $\mathscr{M}_{2}$ has a natural metric induced by the $L^{2}$ inner-product for fields on $\mathbb{R}^{3}$. This metric (see Eq. (2.1) below) was first written down by Atiyah and Hitchin [5], and so has been christened the Atiyah-Hitchin metric. Pursuing Manton's idea we have the following.

Theorem. The Atiyah-Hitchin metric admits closed geodesics.

These geodesics correspond to a static two-monopole rotating with constant angular velocity $\mathbb{M}$. Every static two-monopole has three principal axes, $e_{1}, e_{2}, e_{3}$. (A good description of these axes can be found at the beginning of Chap. 7 of the forthcoming book by Atiyah and Hitchin [6]. Warning: the labelling there of the 2-3 axes is switched relative to ours: their 2-axis is our 3-axis and vice-versa.) $\mathbb{M}$ lies in the $e_{1}-e_{3}$ plane. Its orientation with respect to these axes is depicted in Fig. 1.

* This research was supported in part by the National Science Foundation under grant DMS-87-02502 
Fig. 1

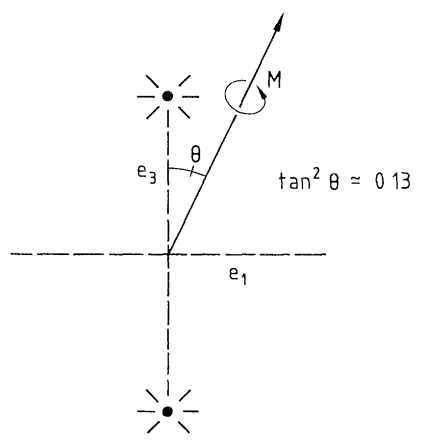

The two radiating dots represent the zeros of the Higgs field. The dotted line connecting them is called the Higgs axis, and is labelled the $e_{3}$-axis in Fig. 1. The angle between the Higgs axis and the angular velocity $\mathbb{M}$ is given by Eqs. (3.3) and (3.4) and is approximately $\pi / 9$. When the Higgs zeros are far apart they can be viewed as the monopole locations: the energy density is concentrated near them. As the distance between the Higgs zeros goes to infinity, rotations about the $e_{3}$-axis becomes symmetries of the field. On the other hand, as the Higgs zeros get close, they can no longer be viewed as the monopole positions, and in fact it is best to give up trying to view the field configuration as that of two distinct "particles." Our geodesics are in this latter regime, as evidenced by the fact that $n_{0}$ of Eqs. (3.1) and (3.2) is close to $\pi$. As the Higgs zeros collide along the $e_{3}$-axis the fields become symmetric with respect to rotations about the $e_{1}$-axis, and their energy density is concentrated near a solid torus of radius approximately $\pi$.

It is still an open problem to decide whether or not these closed geodesics correspond to periodic solutions of the dynamic YMH equations. Our suspicion is that they do.

The proof of the theorem is based upon the fact that the rotation group $S O(3)$ acts on $\mathscr{M}_{2}$ isometrically. The geodesic equations are a system of ordinary differential equations on $T^{*} \mathscr{M}_{2}$. These equations push down to another system of ordinary differential equations, called the reduced equations, on the quotient space $\left(T^{*} \mathscr{M}_{2}\right) / S O(3)$. These equations were first written down by Gibbons and Manton [7]. Wojtkowski [8] used them together with KAM theory to prove that $\mathscr{M}_{2}$ admits bounded quasi-periodic geodesics.

A zero of the vector field defining the reduced equations corresponds to an $S O(3)$ orbit in $T^{*} \mathscr{M}_{2}$ which is invariant under the geodesic flow. Our proof consists of finding such a critical point and then showing that the trajectories on the corresponding $S O(3)$ orbit consists of orbits of one-parameter subgroups of $S O(3)$. The proof of the theorem is then complete since all such subgroups are circles.

\section{The Reduced Equations}

The orbits for the action of $S O(3)$ on $\mathscr{M}_{2}$ are all three-dimensional except for one. This singular orbit consists of the axially symmetric double monopoles (called "collision states" by Atiyah and Hitchin $[5,6]$ and is isometric to $\mathbb{R} P^{2}$, the space of axes in $\mathbb{R}^{3}$. 
The tangent space to a regular orbit may be identified with so(3). Let $\left\{\sigma^{1}, \sigma^{2}, \sigma^{3}\right\}$ be a basis of $s o(3)^{*}$ that is orthonormal with respect to the Killing form. The Atiyah-Hitchin metric on $\mathscr{M}_{2}$ is given by

$$
d s^{2}=f^{2} d \eta^{2}+a^{2}\left(\sigma^{1}\right)^{2}+b^{2}\left(\sigma^{2}\right)^{2}+c^{2}\left(\sigma^{3}\right)^{2},
$$

where $\eta$ is a coordinate transverse to the orbits and the functions $a, b, c$ and $f$ are functions of $\eta$ alone. They are given by

$$
\begin{aligned}
& a^{2}=2 K(K-E)\left(E-K x^{\prime 2}\right) / E, \\
& b^{2}=2 K(K-E) E /\left(E-K x^{\prime 2}\right), \\
& c^{2}=2 K E\left(E-K x^{\prime 2}\right) /(K-E),
\end{aligned}
$$

where $x^{2}+x^{\prime 2}=1$, and

and

$$
K(x)=\int_{0}^{\pi / 2}\left(1-x^{2} \sin ^{2} \phi\right)^{-1 / 2} d \phi,
$$

$$
E(x)=\int_{0}^{\pi / 2}\left(1-x^{2} \sin ^{2} \phi\right)^{1 / 2} d \phi
$$

are the complete elliptic integrals of the first and second kind. In these formulae $x$ is assumed to be a function of $\eta$. We will define this function by

in which case

$$
\eta=2 K(x), \quad \pi \leqq \eta<\infty,
$$

$$
f^{2}=(K-E) E / 2 K\left(E-x^{\prime 2} K\right) .
$$

The singular orbit $\mathbb{R} P^{2}$ corresponds to $\eta=\pi$ (so $x=0$ ), as we have $a(\pi)^{2}=0$. This $\mathbb{R} P^{2}$ is not totally geodesic.

The functions $1 / a^{2}, 1 / b^{2}$, and $1 / c^{2}$ are graphed as functions of $x$ in Fig. 2. Two warnings concerning notation are appropriate. First, our $b$ is called $c$ by Atiyah and Hitchin in [6], and our $c$ is their b. Secondly, the metric Wojtkowski uses in [7] is twice the one we use, (Atiyah-Hitchin's).

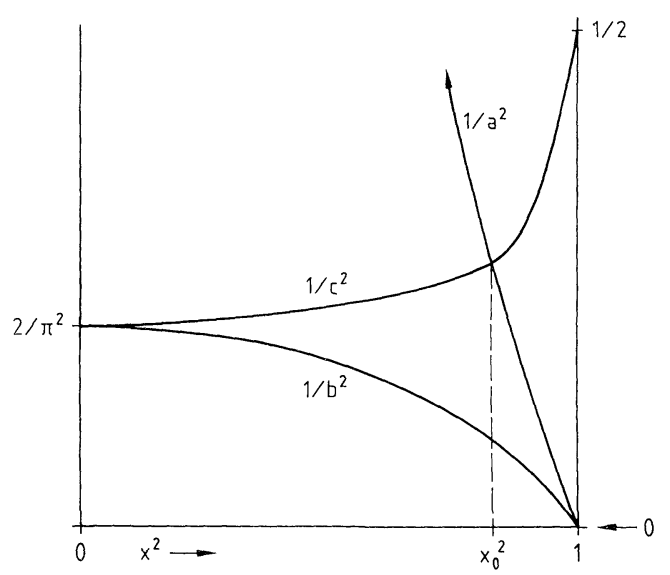

Fig. 2. $x$ is the elliptic modulus. $n_{0}=2 K\left(x_{0}\right)$ in Eqs. (4) and (5) 
The metric (2.1) may be viewed as a symmetric two-tensor on $S O(3) \times[\pi, \infty)$. It is the pull-back of the metric on $\mathscr{M}_{2}$ by a map

$$
\phi: S O(3) \times[\pi, \infty) \rightarrow \mathscr{M}_{2}, \quad \phi(g, \eta)=g \cdot \gamma(\eta),
$$

where $\gamma:[\pi, \infty) \rightarrow \mathscr{M}_{2}$ is a curve in $\mathscr{M}_{2}$ which is perpendicular to the $S O(3)$ orbits and has an endpoint on the singular orbit. We may consider $(g, \eta)$ as a form of polar coordinates for $\mathscr{M}_{2}$.

The equations for geodesic flow are Hamiltonian on $T^{*} \mathscr{M}_{2}$ with Hamiltonian

$$
h=\frac{1}{2}\left(\frac{p^{2}}{f^{2}}+\frac{M_{1}^{2}}{a^{2}}+\frac{M_{2}^{2}}{b^{2}}+\frac{M_{3}^{2}}{c^{2}}\right)
$$

where $p, M_{1}, M_{2}$ and $M_{3}$ are canonically conjugate to $\eta, \sigma^{1}, \sigma^{2}$, and $\sigma^{3}$ respectively. A covector $\alpha$ in these coordinates is written as

$$
\alpha=p d \eta+M_{1} \sigma^{1}+M_{2} \sigma^{2}+M_{3} \sigma^{3} .
$$

The quotient space $T^{*} \mathscr{M}_{2} / S O(3)$ is coordinatized by $(\eta, p, M)$, where $\pi \leqq \eta<\infty$, $p \in \mathbb{R}$, and $M=\left(M_{1}, M_{2}, M_{3}\right) \in \mathbb{R}^{3}$. We further make the identifications $\left(M_{1}, M_{2}\right.$, $\left.M_{3}\right)=\left( \pm M_{1}, \pm M_{2}, \pm M_{3}\right)$, with all the $\pm s$ independent. The quotient space is a Poisson manifold see [9] away from $\eta=\pi$. (At $\eta=\pi$ we have the restriction $M_{1}=0$ which corresponds to the fact that the image of $\sigma^{1}$ blows up as well as further identifications.) The identifications are a result of the isotropy representation. The Poisson brackets are

$$
\left\{M_{i}, M_{j}\right\}=-\varepsilon_{i j}^{k} M_{k}
$$

$\{\eta, p\}=1$, and all other brackets between the coordinates are zero. Hamilton's equations $\dot{f}=\{f, h\}$ are

$$
\begin{aligned}
\dot{M}_{1} & =\left(\frac{1}{c^{2}}-\frac{1}{b^{2}}\right) M_{2} M_{3}, \\
\dot{M}_{2} & =\left(\frac{1}{a^{2}}-\frac{1}{c^{2}}\right) M_{3} M_{1}, \\
\dot{M}_{3} & =\left(\frac{1}{b^{2}}-\frac{1}{a^{2}}\right) M_{1} M_{2}, \\
\dot{\eta} & =p / f^{2}, \\
\dot{p} & =p^{2} f^{\prime} / f^{3}+M_{1}^{2} a^{\prime} / a^{3}+M_{2}^{2} b^{\prime} / b^{3}+M_{3}^{2} c^{\prime} / c^{3},
\end{aligned}
$$

where the "" means $\frac{d}{d t}$ and the "'" means $\frac{d}{d \eta}$. These reduced equations are found in Gibbons and Manton [7, p. 197]. They are also found in Wojtkowski [8].

\section{Proof of the Theorem}

We begin by finding zeros for the reduced vector field. Set $M_{2}=0$ in the reduced equations (2.3). Then $\dot{M}_{1}=\dot{M}_{3}=0$. If we can find an $\eta_{0}$ so that

$$
\frac{1}{a\left(\eta_{0}\right)^{2}}-\frac{1}{c\left(\eta_{0}\right)^{2}}=0
$$


then we have that $\dot{M}_{2}=0$. To see that $\eta_{0}$ exists we note that on the interval $[\pi, \infty)$ the function $\frac{1}{a^{2}}$ decreases monotonically from $\infty$ to 0 , and that $\frac{1}{c^{2}}$ increases monotonically from $\frac{2}{\pi^{2}}$ to $\frac{1}{2}$. It follows that $\frac{1}{a^{2}}-\frac{1}{c^{2}}$ decreases monotonically from $\infty$ to $-\frac{1}{2}$ and hence has a unique zero $\eta_{0}$. We find that

$$
\eta_{0} \cong 4.6 \text {. }
$$

(Alternatively, one calculates that (3.1) is equivalent to the equality $K=2 E$ for the complete elliptic integrals. This implies that the modulus $x$ satisfies $x^{2} \simeq 0.82$.)

Set $p=0$ so that $\dot{\eta}=0$. Finally,

$$
\dot{p}=-\frac{1}{2}\left\{\left(\frac{1}{a^{2}}\right)^{\prime} M_{1}^{2}+\left(\frac{1}{c^{2}}\right)^{\prime} M_{3}^{2}\right\},
$$

so we require that

$$
\frac{M_{1}^{2}}{M_{3}^{2}}=-\frac{\left(1 / c^{2}\right)^{\prime}\left(\eta_{0}\right)}{\left(1 / a^{2}\right)^{\prime}\left(\eta_{0}\right)}
$$

Note that the monotonicity described in the previous paragraph implies that the right-hand side is positive. We calculate this ratio to be approximately 0.13 . This quantity is $\tan ^{2} \theta$, where $\theta$ is the angle between $\mathbb{M}$ and the Higgs axis (see Fig. 1). We find

$$
\theta \simeq 0.35 \simeq \frac{\pi}{9}
$$

We have described a one-parameter family of zeros for the reduced vector field. The parameter is just the length of the covector, so all these critical points represent the same $S O(3)$-orbit of geodesics with a different affine parametrization. Let $z_{0}=\left(\eta_{0}, M_{0}, 0\right)$ denote one such critical point.

It remains to show that these geodesics are closed. Let $\mathbb{M}^{2}=M_{1}^{2}+M_{2}^{2}+M_{3}^{2}$. One calculates that

$$
d h\left(z_{0}\right)=\frac{1}{2} \lambda d\left(\mathbb{M}^{2}\right)\left(z_{0}\right), \quad \text { where } \lambda=\frac{1}{a\left(\eta_{0}\right)^{2}}, \quad \text { a nonzero constant, }
$$

and hence, by pull-back, this same relation holds for any point $\tilde{z}_{0}$ in $T^{*} \mathscr{M}_{2}$ projecting onto $z_{0}$. $\mathbb{M}^{2}$ is the square of the moment map $J: T^{*} \mathscr{M}_{2} \rightarrow \operatorname{so}(3)^{*}$ for the $S O(3)$ action on $T^{*} \mathscr{M}_{2}$. (Warning: The $M_{i}$ are not the components $J_{i}$ of the moment map. In fact, since $J$ is equivariant, as opposed to invariant, its components do not descend to functions on $T^{*} \mathscr{M}_{2} / S O(3)$. However, when the components are taken relative to an orthonormal basis $\left\{e_{i}\right\}$ of $s o(3)$ then it is true that $\Sigma M_{i}^{2}=\Sigma J_{i}^{2}$. The relation between $\mathbb{M}$ and $\mathbb{J}$ is essentially the same as the relation between right trivializing and left trivializing $T^{*} S O(3)$.)

Recall that the Hamiltonian vector field $X_{g}$ of $g$ is defined by $d f \cdot X_{g}=\{f, g\}$. One calculates

$$
X_{\mathbb{M}^{2}}(z)=2 \Sigma J_{i}(z)\left(e_{i}\right)_{T^{*} \mathscr{M}_{2}}=2 \mathbb{J}(z)_{T^{*} \mathscr{M}_{2}},
$$

where we have identified $s o(3)$ and $s o(3)^{*}$ using the Killing form, and have used the notation $\xi_{T^{*} \mathscr{M}_{2}}$ to denote the infinitesimal generator of $\xi \in S O(3)$. Combining these 
results, and recalling that the Hamiltonian vector field $X_{g}$ at a point $z$ of $T^{*} \mathscr{M}_{2}$ depends only on $\operatorname{dg}(z)$, we have

$$
X_{h}\left(\tilde{z}_{0}\right)=\lambda \mathbb{J}\left(\tilde{z}_{0}\right)_{T * M_{2}}, \quad \lambda=\frac{1}{a\left(\eta_{0}\right)^{2}} .
$$

Since $\{h, \mathbb{J}\}=0$, this relation holds along the entire $X_{H}$ orbit through $\tilde{z}_{0}$. Thus this orbit equals the orbit of the one-parameter subgroup generated by $\mathbb{J}\left(\tilde{z}_{0}\right)$. Since all such subgroups are circles, this completes the proof of the theorem.

Future prospects. Our geodesic is unstable. One can see this by linearizing the reduced vector field at an equilibrium $z_{0}$. The eigenvalues of this linearization (restrictred to the kernel of $d\left(\mathbb{M}^{2}\right)\left(z_{0}\right)=0$ ) are of the form $\pm \omega, \pm \bar{\omega}$, where both the real and imaginary parts of $\omega$ are nonzero. It would be interesting to understand the global behaviour of the corresponding stable and unstable manifolds. (These represent sub-manifolds of $T^{*} \mathscr{M}_{2}$ which are invariant under the geodesic flow and which spiral into and out of the geodesic(s) corresponding to $z_{0}$.) In particular are they unbounded? If so, there would be geodesics coming in from infinity which get trapped by our geodesic, or, in the language of Atiyah and Hitchin ([6], end of Chap. 13) the classical geodesic scattering would be incomplete.

It remains to show that near our geodesics there are true solutions to the hyperbolic YMH equations which are time-periodic.

Acknowledgements. This work was partially supported by NSF grant DMS-87-02502. We would like to thank Cliff Taubes for pointing out the interest in closed geodesics on $\mathscr{M}_{2}$. We would also like to acknowledge several clarifying comments by $\mathrm{M}$. Atiyah and by $\mathrm{N}$. Manton on a preliminary version of this note.

\section{References}

1. Jaffe, A., Taubes, C.: Vortices and monopoles. Boston: Birkhäuser 1980

2. Manton, N.S.: Multimonopole dynamics in monopoles in quantum field theory. Singapore: World Science 1982, pp. 87-94

3. Hurtubrise, J.: Monopoles of charge 2. Commun. Math. Phys. 92, 195-202 (1983)

4. Donaldson, S.K.: Nahm's equations and the classification of monopoles. Commun. Math. Phys. 96, 387-408 (1984)

5. Atiyah, M.F., Hitchin, N.J.: Low energy scattering on non-abelian monopoles. Phys. Lett. A 107, $21-25$ (1985)

6. Atiyah, M.F., Hitchin, N.J.: The geometry and dynamics of magnetic monopoles. Princeton University Press, forthcoming 1988

7. Gibbons, G.W., Manton, N.S.: Classical and quantum dynamics of BPS monopoles. Nucl. Phys. B 274, 183-224 (1986)

8. Wojtkowski, M.P.: Bounded geodesics for the Atiyah-Hitchin metric. Bull. A.M.S., 179-185 (1988)

9. Weinstein, A.: The local structure of poisson manifolds. J. Differ. Geom. 18, 523-557 (1983)

Communicated by C. H. Taubes

Received October 22, 1987; in revised form April 15, 1988 